\title{
Les TIC analysées selon une posture féministe en contexte africain
}

ICTs analyzed using a feminist stance in a African context

Joelle Palmieri

\section{(2) OpenEdition}

12 Journals

Édition électronique

URL : http://journals.openedition.org/ctd/1868

DOI : $10.4000 /$ ctd. 1868

ISSN : 2491-1437

Éditeur

Chaire Unesco Pratiques émergentes en technologies et communication pour le développement

Édition imprimée

Date de publication : 12 novembre 2015

Référence électronique

Joelle Palmieri, « Les TIC analysées selon une posture féministe en contexte africain »,

Communication, technologies et développement [En ligne], 2 | 2015, mis en ligne le 11 juin 2019, consulté le 22 juillet 2020. URL : http://journals.openedition.org/ctd/1868; DOI : https://doi.org/10.4000/ctd. 1868

Ce document a été généré automatiquement le 22 juillet 2020

Communication, technologies et développement 


\title{
Les TIC analysées selon une posture féministe en contexte africain
}

\author{
ICTs analyzed using a feminist stance in a African context
}

\author{
Joelle Palmieri
}

1 Au sein de la recherche sur la critique de la «société de l'information", on constate l'expansion du néolibéralisme et l'usage courant des technologies de l'information et de la communication (TIC) par l'Occident ${ }^{1}$ à des fins uniquement économiques, ce qui permet d'expliquer l'existence des inégalités d'accès aux TIC (Chéneau-Loquay, 2002 ; Sagna, 2009). Par ailleurs, l'hypothèse selon laquelle les inégalités de genre sont la seule source des problèmes d'invisibilité politique des organisations de femmes ou féministes dans la « société de l'information » est centrale dans les travaux liés à la problématique « Genre et TIC ( Gurumurthy, 2006 ; Hafkin, 2006 ; Primo, 2003).

Dans ce contexte spécifique, il apparait important d'interroger ces a priori théoriques qui limitent la critique de la «société de l'information» à des questionnements patriarcal, économique et néocolonial ${ }^{2}$. Notre objectif est de renouveler cette critique d'un point de vue féministe. Nous avons choisi d'adopter l'angle particulier, et encore peu examiné, des rapports de domination dont la "société de l'information » serait le produit autant qu'elle en serait productrice.

3 Ce choix incite d'une part à élargir l'investigation vers l'analyse d'autres inégalités que celles entre femmes et hommes. Plus encore, il pousse à dépasser cette notion d' inégalités. Il permet de s'orienter vers celle plus adaptée de domination. En effet, les inégalités, telles qu'elles sont produites dans les sociétés, sont plus largement les instruments de la domination qui régit les relations sociales et en particulier la relation entre institutions et individus. Plus simplement, les inégalités sont le terreau des rapports de domination, de genre, de classe, de «race $»^{3}$ (Dorlin, 2009). En cela, et

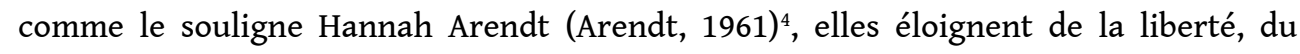
"potentiel», du pouvoir. Adopter ce concept de domination dans la "société de l'information " permet de distinguer domination et pouvoir et ainsi déterminer en quoi les TIC, utilisées ici comme outils de la "société de l'information », peuvent à la fois 
produire des rapports de domination, mais aussi créer des espaces de libération de savoirs non dominés.

4 À partir de la mise en évidence des institutionnalisations croisées des TIC et du genre, l'article s'attache à révéler en quoi la « société de l'information » et les usages des outils qui y sont attachés renouvellent le concept de colonialité, augmentent les facteurs de subalternité, dépolitisent le réel, tout autant qu'ils ouvrent un espace de critique féministe.

\section{Remettre en cause ses méthodes en contexte africain}

5 La domination masculine, le genre, le néocolonialisme, le néolibéralisme sont-ils les bons concepts qui permettent d'établir une analyse critique de la "société de l'information"? Ces concepts rendent-ils compte à eux seuls des rapports de domination dont la "société de l'information» est le produit? Permettent-ils de déterminer que cette "société de l'information» produit elle-même des rapports de domination?

6 Afin de répondre à ces questions, telles qu'elles sont usuellement posées dans le domaine, nous avons rassemblé des résultats qui émanent d'observations participantes réalisées en 2004 dans la province du Cap Occidental en Afrique du Sud auprès de deux organisations de femmes ${ }^{5}$ et entre 2006 et 2008 en Afrique de l'Ouest auprès de trois associations de jeunes ${ }^{6}$. Ces observations portaient respectivement sur des initiatives de récits oraux de vie de femmes et sur des prises de paroles de jeunes sur le genre et la citoyenneté dans le cadre de l'utilisation citoyenne des TIC en vue de l'abandon de l'excision (Palmieri, 2012, p. 173-190).

7 Les résultats découlent également d'entretiens menés en décembre 2008 et en janvier 2009 au Cap en Afrique du Sud et à Dakar au Sénégal auprès de 31 personnes - 15, dont 14 femmes et un homme, âgés de 25 à 60 ans en Afrique du Sud, 16, dont 15 femmes et d'un homme, âgés de 30 à 60 ans au Sénégal. Ces personnes représentaient 24 organisations de femmes ou féministes, instituts de recherche travaillant sur le genre et organisations centrées sur la création numérique, le soutien aux droits des paysans ou à la trithérapie, mais ne travaillant pas dans une perspective de genre. Ces organisations avaient ou non accès à l'Internet, avaient ou non des sites Web. Les entretiens ont reposé sur trois grandes thématiques: (1) interroger les personnes sur leur situation personnelle (lieu de résidence, âge, milieu social, accès à l'Internet...) ; (2) les faire s'exprimer sur le contexte national (situation politique, relations avec le "Nord», situation des femmes, de l'information...) avec un sous-ensemble visant spécifiquement le contexte de la "société de l'information »; (3) les faire se concentrer sur leur support Internet ou leur projet de support, en leur demandant de le qualifier de façon précise : idée de départ, ambition et mission, protagonistes, mise en œuvre, obstacles, processus de recherche et de traitement de l'information, évaluation qualitative et quantitative des usages du support par eux-mêmes et par leurs utilisateurs. Cette enquête a demandé des déplacements sur le terrain. La période à laquelle les entretiens ont eu lieu a correspondu à une situation prélectorale, législative et présidentielle en Afrique du Sud et municipale au Sénégal ${ }^{7}$, qui, même si elle est mentionnée, a paradoxalement eu peu d'incidences sur le contenu des entretiens, qui ciblent pourtant les usages politiques de l'Internet. On aurait en effet pu attendre un engouement dans la description ou l'interprétation de ces usages lié à leur 
hypothétique augmentation pendant les campagnes électorales et les actions qu'elles ont engendrées. Par ailleurs, on a ultérieurement pu observer que la pénétration exponentielle de Twitter ou de médias sociaux privilégiant l'image, que connaît en particulier l'Afrique du Sud depuis le début des années $2010^{8}$ et qui peut autoriser, tel que cela a été observé et analysé (Favero, 2013, 259-277), un dépassement et une transgression des formes de prise de parole dominantes, n'atteint pas encore les organisations interrogées.

Ces entretiens ont été complétés par une approche théorique fournie, selon trois axes : la domination masculine (Delphy, Duerst-Lahti, Kergoat, Guillaumin, Tabet, Héritier, Hirata, Falquet), la société de l'information (Cardon, Proulx, Jouët), la colonialité du pouvoir (Quijano, Grosfoguel, Mignolo). Enfin, une analyse des textes de référence sur les politiques et des études liées à l'intégration du genre dans les programmes internationaux ou nationaux de TIC des deux pays, bornée au corpus des études réalisées par le mouvement "Genre et TIC » et des textes officiels liés à la Conférence de Pékin de 1995 et aux différents Sommets mondiaux de la société de l'information qui se sont tenus depuis 2003, a étoffé le champ d'investigation.

\section{Internet : des enjeux contrastés}

$9 \quad$ Les difficultés d'entente sur les mots utilisés lors des entretiens ont permis de mettre en évidence le peu de sens associé par les acteurs de terrain aux termes institutionnels. Les interlocuteurs dans les deux pays ont exprimé leur faible conscience des " enjeux » de la «société de l'information». La majorité des femmes et leurs organisations utilisent des techniques, infrastructures et logiciels dont elles ne connaissent ni les propriétaires ni les politiques associées.

10 En Afrique du Sud, les TIC, et en particulier l'Internet, ont été considérées comme des sujets déplacés. Selon Mercia Andrews, directrice d'une ONG spécialiste des droits des paysans, l'Internet fait écho à « toutes ces choses techniques que le gouvernement a essayé d'implanter sous forme de centres de télécommunication [...], mais qui n'a pas marché ». Helga Jansen, journaliste, a parlé d'« outil de travail» peu adapté à la majorité des femmes qui rencontrent des difficultés à "écrire ce qu'elles ressentent ». Helga a insisté sur la vision biaisée de la réalité que véhicule l'Internet, vision qui traduit une division de classe, puisque les messages diffusés ne peuvent l'être que par des gens qui ont les moyens de le faire. Argument repris par Liesl Theron, qui dirige une ONG de défense des droits des LGBT $^{9}$ et a affirmé que l'Internet ne concerne pas les femmes qui vivent dans « des cabanes et ont à partager des toilettes communes avec cinquante autres familles " et cherchent seulement à avoir accès à l'eau et à la nourriture. L'Internet est alors entendu comme réservé à celles qui en ont les moyens. Son accès est cher et la priorité est davantage à la lutte contre l'aggravation des écarts de richesse, du chômage, de la pauvreté, contre l'accélération des violences sexuelles et politiques, contre l'affaiblissement des mouvements sociaux, y compris féministes.

$11 \mathrm{Au}$ Sénégal, c'est l'utilisation du terme «féminisme» qui a fait blocage. Les interlocuteurs ont plus volontiers utilisé les termes «droits des femmes » ou « genre ». Par exemple, Aminata Kébé, juriste, coordinatrice d'une association nationale qui dispense des conseils juridiques aux femmes et qui alimente un site Web sur les droits des Sénégalaises, a souligné : « on est plus préoccupé par la promotion et la protection des droits des femmes, [par le fait] que ce site [web] soit accessible à toutes les femmes, 
pas aux femmes intellectuelles seulement, à toutes femmes qui arrivent à se connecter, pour qu'elles puissent avoir une information ou plus, à défaut d'avoir l'information, d'avoir un contact pour pouvoir appeler, pour demander conseil, renseignement, information ». En revanche, l'usage des TIC pour le genre ou pour l'égalité a suscité un engouement quasi général. L'évocation du mot TIC permet de rendre compte du lien entre pauvreté et résistance, violences et détermination, discriminations sexistes et justice, dette et informalité. Fatou Diop, présidente du Conseil sénégalais des femmes (COSEF), a parlé de la possibilité d'avoir "assez d'informations pour pouvoir agir, proposer, penser ». Fatou Ndiaye Turpin, coordinatrice d'un important réseau national de dix-sept organisations de femmes, a parlé d'obtenir des informations « de manière instantanée » et pour pas cher (moins que le téléphone).

12 En dehors de cette distance à la rhétorique institutionnelle en Afrique du Sud et au Sénégal, des singularités entre les deux pays ont émergé. Elles se sont installées notamment dans les rapports au pouvoir politique et aux journalistes. Les Sud-Africains situent majoritairement le droit à l'information au niveau constitutionnel, donc en relation avec l'État, alors que la plupart des Sénégalais confient ce droit aux journalistes, pour leur grande majorité du secteur privé. À la question «Quelle est la place des libertés d'expression, de circulation, des droits en général ?", Madjiguéne Cissé, présidente du Réseau des femmes pour le développement durable en Afrique au Sénégal, a répondu «ils ont saccagé des locaux de journaux, ils ont emprisonné des journalistes, etc., mais n'empêche que quand même, relativement, la presse se prononce assez librement, les gens aussi peuvent se prononcer à travers les médias, dire ce qu'ils pensent, même sur le gouvernement ». Sally-Jean Shackleton, directrice d'une organisation spécialiste des questions «Genre et TIC » en Afrique du Sud, a affirmé quant à elle: «Je pense que nous avons le droit à la libre expression, nous avons une très bonne constitution ». Dans les deux cas, ce sont ces intermédiaires l'État, les médias -, et non les interlocuteurs eux-mêmes, qui seraient directement concernés par ce droit et par le pouvoir qu'il confère.

L'information ne semble ni être considérée comme un droit ni comme un outil stratégique d'action ou de mobilisation qu'elle soit sociale ou politique. La majorité des organisations de femmes ou féministes consultées ne s'en sentent pas propriétaires. Elles vivent leur dépendance envers les intermédiaires qu'elles citent avec une assez bonne tolérance et la considèrent comme suffisamment efficace.

14 La différence entre organisations féministes et féminines, quel que soit le pays, est étonnamment peu perceptible dans le domaine. On assiste globalement à un discours généraliste et basique sur les usages d'Internet: même si les TIC sont éloignées de la réalité quotidienne des femmes, elles demeurent paradoxalement un bon moyen de « lutte », de « promotion des droits", de " recueil d'informations».

En revanche, la "fracture numérique », terminologie adoptée par la majorité des interlocuteurs, ouvre à différentes interprétations au sein même de chaque pays. Elle permet de distinguer trois groupes : ceux qui savent comment aller au-delà et ont des idées sur ce qu'il faudrait mettre en œuvre pour y faire face, ceux qui ne savent pas la combattre et ceux qui reconnaissent leur ignorance, mais aussi leur dépendance du fait de cette ignorance, qui les éloignent de solutions viables et à long terme, y compris sur leurs terrains de lutte.

L'ensemble de ces résultats va demander à être confronté avec l'analyse des textes institutionnels telle qu'elle a été menée. 


\section{Deux institutionnalisations croisées}

16 programmes de TIC a permis, tout d'abord, de constater un phénomène temporel intéressant : l'émergence de la "société de l'information » coïncide avec la quatrième Conférence mondiale sur les femmes organisée par l'ONU à Pékin en 1995. À cette date, les États, en collaboration avec les entreprises du secteur des télécommunications et de l'informatique, ont formulé des politiques de TIC et, concomitamment, ont élaboré des politiques d'égalité de genre. Les textes forcent le constat que les politiques de TIC sont venues influencer les politiques d'égalité de genre. En effet, dans la Plate-forme d'action de Pékin, adoptée le 15 septembre 1995 lors de cette Conférence mondiale, on peut lire que de "nouvelles possibilités » sont offertes par «l'informatique et la télévision par satellite et par câble » en matière de "participation des femmes aux communications et aux médias, ainsi que pour la diffusion d'informations sur les femmes $»^{10}$. Ce texte ratifié par les représentants de 186 pays va incarner le point de départ de la mobilisation de différents acteurs autour d'un nouveau concept, la "fracture numérique de genre ", qui sera dès lors régulièrement repris dans les discours officiels et dans l'ensemble des mécanismes de l'institutionnalisation des TIC.

À partir de ce texte de référence, de nombreuses études concernant notamment l'Afrique ont vu le jour, menées pour la plupart par les militantes du mouvement «Genre et TIC » (Gurumurthy, 2004). En particulier l'étude d'Enda Tiers-Monde de 2005 sur la fracture numérique de genre en Afrique de l'Ouest (Enda, 2005, p. 64) montre que les aspects « sérieusement préoccupants » en matière d'inégalités de genre dans les TIC sont le contrôle et le contenu, comparativement aux aspects connectivité ou capacités ${ }^{11}$. Par ailleurs, selon une recherche menée conjointement par l'Association of Progressive Communicators-Africa-Women et par Femnet en 2000 (Lowe \& Khan, 2000) plus de $70 \%$ des ordinateurs hôtes qui forment les fondations de l'Internet sont installés aux États-Unis ; l'anglais est utilisé dans près de $80 \%$ des contenus des sites Web bien que moins de dix pour cent des personnes dans le monde parle cette langue ${ }^{12}$. Le pourcentage des contenus en anglais a certes diminué et est passé à $45 \%$ en 2005, ${ }^{13}$ mais comme Daniel Prado (Prado, 2012), Secrétaire exécutif du Réseau mondial pour la diversité linguistique, Maaya, le souligne "Une chose est certaine, l'anglais reste la langue la plus utilisée sur internet ». Si bien que l'Afrique génère environ $0,4 \% \mathrm{du}$ contenu global, $0,2 \%$ en excluant l'Afrique du Sud. Sur ces $0,2 \%$ de contenus africains, moins du tiers sont produits par des femmes (Lowe \& Khan, op. cit.). Ce qui fait chuter le pourcentage de contenus créés par des Africaines dans le monde à $0,07 \%$. Ruth Ochieng de Isis-Wicce confirme cette faible contribution des Africaines: «les femmes en Afrique n'impactent toujours pas ce qui est jugé comme une "connaissance globale acceptable". Cette anomalie révèle l'hégémonie de certains groupes dans la diffusion et la production de connaissance [qui] résulte de la monopolisation de la sphère du "savoir global" " (Ochieng, 2002, p. 3). En effet, la majorité des contenus (texte, audio, vidéo...) diffusés au moyen des TIC est globalement écrite par des hommes et surtout diffusée par des hommes ${ }^{14}$. L'écriture au quotidien sur des blogs, dans des séances de clavardage (chat) et d'autres galeries virtuelles, telle qu'elle est plus aisément pratiquée en Amérique du Nord et en Europe de l'Ouest, demeure en Afrique le fait d'une élite ${ }^{15}$. En termes de création numérique, ce sont majoritairement des hommes, et, en particulier, des hommes 
blancs, qui programment des logiciels, y compris dans le mouvement des logiciels libres (Ghosh \& alii, 2002). En termes de contrôle, les femmes sont sous-représentées dans toutes les structures de décision du secteur des TIC et ont relativement peu de contrôle et d'influence sur les processus de prise de décision (Marcelle, 2005). Les propriétaires des entreprises privées de télécommunication sont majoritairement des hommes ${ }^{16}$. productrice d'inégalités entre les genres en son sein : «Le fossé qui sépare les sexes est, de toutes les inégalités celle que la révolution numérique a le plus amplifiée, car elle traverse toutes les couches sociales et économiques ». Aussi, la question n'est pas tant la difficulté de l'accès aux ressources des TIC ou aux formations afférentes, mais la rémanence des relations sociales et économiques inégalitaires existantes. On assiste ainsi à une reproduction par les TIC des inégalités existantes.

Pourtant, les organisations en charge des politiques de TIC continuent à expliquer les inégalités de genre portées par les TIC en utilisant cette formule, la "fracture numérique de genre », et en se figeant sur les capacités et la connectivité. Les actions entreprises, tant par les pouvoirs publics, les organisations internationales ou les organisations de la société civile sénégalaises et sud-africaines inscrites dans une politique d'appropriation citoyenne des TIC, convergent vers :

- des politiques d'accès aux infrastructures, notamment par des campagnes de sensibilisation proposant aux femmes et aux jeunes des formations adaptées,

- et l'accès à des lieux, principalement des cybercentres, cybercafés ou lieux communautaires, à coût réduit, dans l'espoir que les personnes ciblées mettent en application les connaissances acquises.

20 On peut en déduire que l'institutionnalisation du genre dans les TIC a pour ambition majeure d'être économiquement efficace (développement des infrastructures de télécommunications, des commerces liés aux usages d'internet, des formations, etc.). À ce titre, elle introduit un biais dans la notion d'inclusion sociale, par exemple. Nous allons maintenant analyser comment, et de surcroît en quoi les TIC sont également productrices d'inégalités.

\section{Le genre facteur de subalternité}

21 Les ambitions de l'institutionnalisation ne sont pas uniquement économiques. La question de "rendre les femmes autonomes, lutter contre la mortalité infantile, améliorer la santé des mères », en particulier en Afrique, telle qu'elle apparaît dans la Déclaration de principe du Sommet mondial de la Société de l'Information de 2004 intitulée "Construire la société de l'information: un défi mondial pour le nouveau millénaire » (UIT, 2004), sous-entend que ces femmes sont prises comme des personnes à soutenir, à assister et à éduquer, et qu'elles forment un groupe homogène. On peut pourtant se demander si elles sont riches ou pauvres, noires, métisses ou blanches, jeunes ou moins jeunes, urbaines ou rurales, mariées ou non, mères de famille ou non, hétérosexuelles ou homosexuelles, lettrées ou illettrées, en besoin ou non d'être connectées... Ne pas se poser cette question et intégrer ce non-pensé dans les politiques internationales devient une question d'intrusion au quotidien du virtuel sur les modes de pensée et d'agir des femmes. 
22 Par l'institutionnalisation du genre dans les TIC, «les femmes», en tant que groupe social, se retrouvent placées au rang de victimes ou d'actrices immobiles, ayant besoin d'encadrement technique, d'assistance, de soutien financier, parce que plus affectées par la pauvreté ou par le manque d'éducation par exemple. Leurs savoirs propres ne sont pas pris en compte. Comme le souligne Gayatri Spivak (Spivak, 1988a), ces femmes, qui n'ont pas droit à la parole, en viennent à gérer au pied levé ce qui est globalement et socialement considéré comme périphérique (en particulier la gestion du quotidien). Elles sont à ce titre ce que la philosophe indienne nomme des «subalternes » et leur subalternité est renforcée, car elles ne sont potentiellement pas connectées par manque de savoirs adaptés aux TIC (informatique, internet, etc.).

23 En outre, il existe une frontière entre les TIC et les "autres" thèmes de luttes de femmes ou féministes. Rita Edwards, directrice d'un centre sud-africain de promotion du genre témoigne : «la fracture numérique est davantage à l'ordre du jour de nos dirigeants, nos gardiens. Nous, nous sommes confrontées aux écarts de richesse, au chômage... ". En Afrique, les expressions des femmes, leurs contenus ne peuvent être mis en lumière selon la priorité qu'elles souhaitent. Ils sont dominés par les savoirs de ceux qui décident des politiques de TIC, par définition importés, majoritairement d'Occident. Les priorités de genre concernant les TIC sont définies par les cadres des institutions internationales et non par les organisations dites «bénéficiaires " elles-mêmes. Ces dernières sont alors invitées notamment par leurs bailleurs à hiérarchiser leurs activités/mobilisations et notamment à intégrer la lutte conte «la fracture numérique» dans leurs actions, alors que telle n'est pas leur priorité. Les institutionnalisations conjointes des TIC et du genre participent alors de l'invisibilité des sujets des luttes des organisations de femmes africaines.

24 Ainsi, dans la "société de l'information", le genre participe de la subalternité des femmes africaines. Si on ajoute à ce constat l'analyse précédemment effectuée de leurs usages des TIC, on peut affirmer que la mise en exergue des inégalités et identités de genre est désormais liée aux TIC.

\section{Une colonialité renouvelée}

Les analyses mobilisées jusqu'ici ont montré que les «inégalités de genre " ne se mesurent plus uniquement aux aspects économiques ou infrastructurels de la "société de l'information». Aussi il semble nécessaire de dépasser cette première analyse critique et de creuser l'aspect épistémique de ces impacts, c'est-à-dire l'aspect hiérarchisé des savoirs qui traversent ce système.

Nous cherchons à déterminer si la « société de l'information » s'apparente à une société basée sur des outils et des réseaux dans laquelle des enjeux de pouvoir se produisent et se reproduisent.

Les interlocuteurs ont été interrogés à la fois sur la façon de maintenir le site web - ils sont peu autonomes et sont majoritairement dépendants d'un sous-traitant informatique -, sur les contenus publiés et la façon de les concevoir, sur l'usage du site web comme outil de mobilisation et sur leur audience. Il ressort des entretiens une triple posture, commune aux deux pays : le peu d'intérêt porté à l'avis de l'internaute, le présupposé selon lequel l'internaute ne peut rien apporter à l'organisation, et par voie de conséquence, le site Web conçu comme une force de vente de l'organisation 
auprès de ceux qui ont les moyens de la financer. Ce commun corrobore le peu d'attention portée aux cibles du site Web et à leurs usages. Les organisations publient pour publier sans se soucier de savoir si les contenus sont lus, ni davantage de qui les lit, ou prétendent connaître en amont leurs lecteurs. Le site Web ne répond pas aux besoins de supposés lecteurs, mais plutôt aux exigences des bailleurs de l'organisation.

Réclamés par ces bailleurs, indispensables à la survie des organisations, les usages des TIC sont devenus une norme, un passage obligé vers une nécessaire modernité. Fatimata Seye Sylla, présidente d'une association sur l'éducation des jeunes par les TIC au Sénégal, est formelle : «Les bailleurs de fonds ne financent pas l'idée de quelqu'un. Pour être financé, il faut voir ce qu'ils veulent ! Faire et créer un projet en fonction de ça ». Aussi a-t-elle obtenu des financements parce qu'elle a répondu à un programme d'un de ses bailleurs sur la "lutte contre la fracture numérique, qui appuient des télécentres, qui font de la formation, etc. ». Aussi, les usages ne sont pas adaptés au contexte des actions des organisations, mais à celui des programmes des bailleurs. Madjiguéne Cissé témoigne : «L'Internet est efficace pour le milieu urbain, mais reste difficile en milieu périurbain ou rural. Nous transmettons les rapports d'activité par les chauffeurs qui prennent les enveloppes et les distribuent sur leur route ». Ou encore Rowayda Halim, présidente d'une organisation féministe sud-africaine : "Seules $5 \%$ des femmes savent se servir d'ordinateur en Afrique du Sud, le même taux que les personnes en mesure de recevoir de l'information. Le support de communication le plus efficace est désormais le téléphone mobile et le SMSism ${ }^{17}$, même s'il reste inadapté pour les illettrés ».

De fait, l'introduction des usages de l'informatique, des télécommunications et de la communication en réseaux est, dans les deux pays de l'étude, généralisée. Cette généralisation influe sur la gestion de la vie quotidienne, socialement dévolue aux femmes, car elle bouleverse, comme en ont témoigné les interlocuteurs, le rapport des individus sociaux au temps et à l'espace. Mercia Andrews confirmera : « Nous avons des difficultés d'accès aux ordinateurs, aux compétences, à l'électricité, au réseau sans fil, toutes ces choses techniques que le gouvernement a essayé d'implanter sous forme de centres de télécommunication en zones rurales, mais qui n'ont pas marché. Concernant les femmes, c'est bien pire, car, dans les zones rurales, dans la plupart de nos townships, ce sont les jeunes femmes qui rentrent à la maison après l'école et qui doivent s'occuper de leurs frères et sœurs, nettoyer la maison, faire la cuisine, faire attention aux enfants, au point qu'elles n'ont pas de temps supplémentaire pour apprendre à utiliser un ordinateur ».

La lecture d'Anibal Quijano (Quijano, 1994) permet alors d'associer un concept à la "société de l'information »: colonialité du pouvoir. Tel que le sociologue péruvien la définit, en caractérisant l'ensemble des relations sociales produites par l'expansion du capitalisme en ses périphéries subalternes (ibid.), la "colonialité du pouvoir» s'applique à la "société de l'information » qui accélère, automatise, étend, augmente cette expansion par machines, logiciels et télécommunications interposés. Aussi, audelà de l'analyse de la production d'inégalités de genre, il semble pertinent de préciser le contexte dans lequel ces TIC sont utilisées; depuis leur naissance, on assiste à la mondialisation des rapports sociaux, tout autant économiques, que politiques et épistémiques (Palmieri, 2014, p. 85-108). On connait une forme spécifique et historicisée des rapports de domination entre États et sociétés, ce qui définit pleinement la colonialité du pouvoir (op. cit.). 
31 Forte de cette grille d'analyse conceptuelle, on peut affirmer que la «société de l'information » impacte les actions des organisations de femmes, en particulier en Afrique, renouvelle la colonialité du pouvoir et s'apparente davantage à ce que nous nommerons désormais une société numérique colonialitaire (qui se rapporte à la colonialité).

\section{Vers la dépolitisation du réel}

32 Dans cette société numérique colonialitaire, les actions politiques des organisations de femmes et féministes africaines se négocient entre financement et intervention locale, oscillent entre marchandisation - pour répondre à l'action politique des bailleurs et organisations internationales, de l'Amérique du Nord et de l'Europe de l'Ouest - et mobilisation politique (par rapport à leur État) (Palmieri, 2011, p.115-117). L'usage des outils numériques devient un besoin à satisfaire dans les relations sociales et plus particulièrement dans l'action politique; il crée parfois une obligation, ce qui crée mystification. Cette mystification repose sur l'amalgame entre outils, logiciels et usages, sans plus de nuances sur les effets différenciés des uns et des autres. Elle demande à analyser de façon distincte les actions dans le réel, là où se gère le quotidien et celles dans le virtuel. Par exemple, est-ce le mobile en tant que machine qui agit sur le réel ou est-ce son usage ? Est-ce Facebook qui fait les révolutions, ou est-ce que ce sont les personnes qui l'utilisent pour rendre compte des actes de répression d'une manifestation à laquelle elles ont participé ? Cette manifestation n'a-t-elle pas été convoquée par des groupes organisés en amont dans le réel? Pourquoi, dans leur grande majorité les organisations de femmes interrogées dégroupent virtuel et réel, genre et TIC ? Pourquoi alimentent-elles ainsi un système de hiérarchisation de l'action politique? En ne prenant pas en compte à la fois ce flou et cette opposition entre virtuel et réel, nous négligeons la potentielle dépolitisation du réel par le virtuel.

\section{Les TIC : espace critique féministe?}

Cette hypothèse pousse à explorer trois pistes de réflexion pour la pensée critique féministe $-1^{\circ}$ ) repartir du vécu quotidien réel (Bennett, 2008), $2^{\circ}$ ) requalifier le genre à sa juste valeur par l'analyse des inégalités liées aux multiples identités dans un contexte mondialisé qui renforce la hiérarchisation des pouvoirs (McFadden, 2000), et $3^{\circ}$ ) fouiller des stratégies de contournement de l'expression publique de la domination masculine via le privé, l'intime, l'invisibilité, stratégies que nous avons pu observer en Afrique du Sud et en Afrique de l'Ouest entre 2004 et 2008. Ces expériences africaines placent en effet les savoirs libérés au niveau de leurs auteurs et non de leurs interprètes et resituent l'intime et le privé, au cœur de la problématique des rapports de pouvoir. Les TIC jouent alors une partition expérimentale qui apparait intéressante de lire et d'étudier point par point (Palmieri, 2012, p. 182-186).

Par leurs caractères non formels, non codifiés, non normés, non revendiqués, non installés, non institutionnalisés, ces savoirs transgressent le système tel que nous venons de le décrire. Pour exister, ils rompent avec les méthodologies de recueil de témoignages ou d'observation, voire d'entretiens. Ils s'écartent par ailleurs des théories et pratiques classiques de la communication. Ces savoirs créent les bases d'une communication informelle où les actrices deviennent des auteures. Elles y libèrent leur 
propre champ de connaissance. Elles ouvrent des pistes de renouvellement d'une réflexion féministe sur les relations sociales, sur l'observation de ces relations et sur leurs méthodologies d'analyse.

\section{Vers une épistémologie renouvelée de la domination}

En définitive, la « société de l'information » est le théâtre de deux institutionnalisations synchronisées et concomitantes : le genre et les TIC. La multiplicité des "inégalités " développées en son sein montre que l'on ne saurait se limiter au concept d'« inégalité » et qu'il est nécessaire de développer une critique basée sur les rapports de domination. L'étude, basée sur une enquête, une approche théorique et une analyse des textes institutionnels des organisations internationales, fait apparaitre des pistes pour repenser la colonialité. Loin d'un système basé sur des relations sociales régies par des inégalités économiques et sociales, ce qu'on peut désormais nommer la société numérique colonialitaire alimente, reproduit et produit des rapports de domination. Cette re-production fait apparaître une aggravation de la subalternisation des femmes africaines par genre interposé et une dépolitisation des luttes des organisations de femmes africaines par le virtuel.

Les TIC et le genre sont des variables qui ne peuvent être séparées pour analyser les rapports de domination sous peine de ne pas identifier les espaces d'innovation de la subalternité. L'accélération et la cristallisation des rapports de pouvoir permettent la maturation d'une épistémologie renouvelée de la domination.

\section{BIBLIOGRAPHIE}

Éléments bibliographiques

Arendt Hannah, Condition de l'homme moderne, Poche, Paris, 1961.

Arendt Hannah, "Sur la violence ", in Du mensonge à la violence. Essais de politique contemporaine, traduction française, Calman-Lévy, Paris, 1972, p. 105-208 [on violence, édition originale en anglais 1970].

Bennett Jane, « Researching for Life: Paradigms and PowerEditorial », Feminist Africa $n^{\circ} 11,2008$. Disponible en ligne : <http://agi.ac.za/sites/agi.ac.za/files/fa_11_3_editorial.pdf>, consulté le 25 juillet 2012.

Cardon Dominique, La Démocratie Internet, Le Seuil, Collection Sciences humaines/Divers, Paris, 2010.

Cheneau-Loquay Annie, « Défis liés à l'insertion des technologies de l'information et de la communication dans les économies africaines. L'exemple d'Internet au Sénégal », in Djeflat Abdelkader \& Boindin Bruno, Ajustement et technologie en Afrique, Publisud, Paris, 2002, p. 103.

Davis Angela, Femmes Race et Classe, Des Femmes Paris, 1983. 
Delphy Christine, L'ennemi principal : 1. Économie politique du patriarcat, Syllepse, Paris, 1998.

Delphy Christine, L'ennemi principal :2. Penser le genre, Syllepse, Paris, 2001.

Dorlin Elsa (dir.), avec la collaboration d'Annie Bidet-Mordrel, Sexe, race, classe : pour une épistémologie de la domination, Puf, coll. Actuel Marx/Confrontations, Paris, 2009.

Duerst-Lahti Georgia et Mae Kelly Rita, Gender Power, Leadership, and Governance, University of Michigan Press, 1996.

Enda, « Fracture numérique de genre en Afrique francophone : une inquiétante réalité ", Études et Recherches, $\mathrm{n}^{\circ} 244$, Réseau genre et TIC, Enda, Dakar, 2005, p. 15. Disponible en ligne : <http:// smsi.francophonie.org/IMG/pdf/fracturenumeriquedegenre.pdf>, consulté le 21 juin 2012.

Falquet Jules, «"Genre et développement" : une analyse critique des politiques des institutions internationales depuis la Conférence de Pékin ", Les Pénélopes, 2003. Disponible en ligne : <http:// penelopes.org/xarticle.php3?\%20id_article=3358>, consulté le 4 mars 2010.

Favero Paolo, « Getting our hands dirty (again): Interactive documentaries and the meaning of images in the digital age », Journal of Material Culture, septembre 2013, Vol 18, $\mathrm{N}^{\circ} 3,2013$, p. 259-277.

Ghosh Rishab A. \& alii, Part 4 : Survey of Developers, In Free/Libre and Open Source Software: Survey and Study, International Institute of Infonomics University of Maastricht, The Netherlands. 2002. Disponible en ligne : <http://flossproject.org/report/FLOSS_Final4.pdf>, consulté le 18 mai 2011. Grosfoguel Ramon, «Les implications des altérités épistémiques dans la redéfinition du capitalisme global - Transmodernité, pensée frontalière et colonialité globale », in Multitudes, III (26), Mineure : Empire et « colonialité du pouvoir », 2006, p. 51-74.

Guillaumin Colette, « Race et nature : Système des marques, idée de groupe naturel et rapport sociaux », in Pluriel, n 11, 1977, p. 39-55.

Guillaumin Colette, Sexe, race et pratique du pouvoir. L'idée de Nature, Côté-femmes, Paris, 1992 (1978).

Gurumurthy Anita, Gender and ICTs: Overview Report, Bridge, Londres, 2004. Disponible en ligne : <http://www.bridge.ids.ac.uk/reports/cep-icts-or.pdf>, consulté le 14 mai 2011.

Gurumurthy Anita, « Promoting gender equality? Some development-related uses of ICTs by women, Development ", in Practice, XVI (6), novembre 2006, Routledge, 2006. Disponible en ligne : <http://www.siyanda.org/docs/gurumurthyicts.pdf>, consulté le 14 mai 2011.

Hafkin Nancy J. et Huyer Sophia (dir.), Cinderella or Cyberella?: Empowering Women in the Knowledge Society, in Gillian Youngs, Development in Practice, Vol. 17, No. 3 (juin 2007), p. 463-465.

Harding Sandra, Whose Science? Whose Knowledge? Thinking from Women's Lives, Cornell University Press, Ithaca, New York, 1991.

Héritier Françoise, Masculin, Féminin. La pensée de la différence, Odile Jacob, Paris, 1996.

Hirata Helena et alii (dir.), Dictionnaire critique du féminisme, Puf, ( $2^{\mathrm{e}}$ édition augmentée 2004), Paris, 2000.

Jouët Josette, « Technologies de communication et genre : des relations en construction ", in Réseaux, $\mathrm{n}^{\circ} 120,2003$, p. 53-86.

Kergoat Danièle, «La division du travail entre les sexes », in Kergoat Jacques (dir.), Le monde du travail, La Découverte, Paris, 1998, p. 319-327. 
Morna Colleen Lowe et KhanZohra, Net gains: African women take stock of information and communication technologies, recherche de l'Association of Progressive Communicators-AfricaWomen \& Femnet, Gender Links, Johannesbourg, 2000, 107 p., p. 52. Disponible en ligne : <http:// www.apc.org/fr/system/files/ Net\%20Gains\%20Women\%20in\%20Africa\%20Take\%20Stock\%20of\%20ICTs.pdf>, consulté le 30 novembre 2013.

Marcelle Gillian, « Thinking BIG to Accelerate Gender Equality and Transformation in the ICT Arena ", in Ng Cecilia et Mitter Swasti (dir), Gender and the Digital Economy - Perspectives from the Developing World, Saga Publications, 2005, p. 231-252.

McFadden Patricia, Issues of Gender and Development from an African Feminist Perspective, contribution présentée en l'honneur de Dame Nita Barrow, au Centre des Études en Genre et développement, University of the West Indies, Bridgetown, Barbados, novembre 2000. Disponible en ligne : <http://www.escueladefeminismo.org/spip.php?article153>, consulté le 30 mars 2010.

Mignolo Walter, Local histories, global designs. Coloniality, subaltern knowledge and border thinking, Princeton University Press, New Jersey, 2000a.

Mignolo Walter, « Rethinking the colonial model », in Valdés Mario J. et Linda Hutcheon (dir.), Rethinking literary history, Oxford University Press, Oxford, 2000b, p. 155-193.

Mignolo Walter, « Coloniality at large: time and the colonial difference », in Rodriguez Larreta E. (dir.), Making and Possible Futures, Unesco \& Instituto de Pluralismo Cultural, Rio de Janeiro, 2000c, p. 237-272.

Ochieng Ruth, "Gender Research/Teaching Forum. Information and Communication Technologies as a Tool for Women's Empowerment and Social Transformation, in Africa", in Feminist Africa, Intellectual Politics, $\mathrm{n}^{\circ}$ 1, 6 p., 2002, p. 2. Disponible en ligne : <http:// www.feministafrica.org/index.php/information-and-communication-technologies>, consulté le 24 octobre 2009.

Palmieri Joelle, « Les TIC, outils des subalternes ? », in Kungua Benoît Awazi Mbambi (dir), Leadership féminin et action politique

Le cas des communautés africaines du Canada, Actes du colloque annuel du Centre de Recherches Pluridisciplinaires sur les Communautés d'Afrique noire et de diasporas (CERCLECAD), en collaboration avec le Laboratoire d'Études Africaines et Diasporiques (LEAD) de l'Université d'Ottawa, 15 décembre 2012 à Ottawa (Canada), L'Harmattan, Paris, janvier 2014, p. 85-108.

Palmieri Joelle, «Les femmes non connectées : une identité et des savoirs invisibles ", in Joubert Lucie (dir.), 2012, Les voix secrètes de l'humour des femmes, Revue Recherches féministes, numéro 25,2 (novembre 2012), Québec, 2012, p. 173-190.

Palmieri Joelle, «Technologies : produits du pouvoir et productrices d'impuissance », in From cyborgs to Facebook, technological dreams and feminist critiques, Revue Sophia actes du colloque d'octobre 2011, Sophia, Bruxelles, juin 2012, p. 108-127.

Prado Daniel, « Présence des langues dans le monde réel et le cyberespace », in Vannini Laurent \& Le Crosnier Hervé, Net.lang : Réussir le cyberespace multilingue, C\&F Éditions, Paris, 484 p., 2012, p. 39. Disponible en ligne : <http://net-lang.net>, consulté le 6 novembre 2012.

Primo Natacha, Gender issues in the Information Society, Unesco, Paris, 2003. Disponible en ligne : $<$ http://portal.unesco.org/ci/en/file_download.php/

250561f24133814c18284feedc30bb5egender_issues.pdf>, consulté le 11 mars 2009.

Proulx Serge, « Trajectoires d'usages des technologies de communication : les formes d'appropriation d'une culture numérique comme enjeu d'une société du savoir », in Annales des 
télécommunications, tome 57, n 3-4, 2002. Disponible en ligne : <http://sergeproulx.uqam.ca/wpcontent/uploads/2010/12/2002-proulx-trajectoires-d-57.pdf>, consulté le 18 mai 2011.

Proulx Serge, La révolution Internet en question, Québec-Amérique, Montréal, 2004.

Proulx Serge, « Penser les usages des technologies de l'information et de la communication aujourd'hui : enjeux - modèles - tendances », in Vieira Lise et Pinede-Wojciechowski Nathalie, Enjeux et usages des TIC : aspects sociaux et culturels, Tome 1, Presses universitaires de Bordeaux, 2005, p. 7-20.

Quijano Anibal, «Colonialité du pouvoir et démocratie en Amérique latine », in Multitudes « Amérique latine démocratie et exclusion, Quelles transitions à la démocratie? ", juin 1994. Disponible en ligne : <http://multitudes.samizdat.net/Colonialite-du-pouvoir-et>, consulté le 23 septembre 2008.

Sagna Olivier, Application du triptyque " privatisation, libéralisation, régulation » au secteur des télécommunications. Bilan de l'expérience sénégalaise, communication présentée au Symposium Netsuds à Bordeaux en France, 13-16 octobre 2009. Disponible en ligne : <http://www.gdrinetsuds.org/IMG/pdf/Sagna-2.pdf>, consulté le 9 février 2010.

Saïd Edward W., L'Orientalisme. L'Orient créé par l'Occident, Le Seuil (Parution originale 1980), Paris, 1997.

Spivak Gayatri C., Can the Subaltern Speak? (Les Subalternes peuvent-illes parler ?, traduction française de Jérôme Vidal, Amsterdam, Paris, 2006), in Nelson Cary et Grossberg Lawrence (dir.), Marxism and the Interpretation of Culture, University of Illinois Press, Chicago, 1988a, p. 271-313.

Spivak Gayatri C., In Other Worlds: Essays in Cultural Politics, Routledge, New York, $1988 \mathrm{~b}$.

Tabet Patricia, La construction sociale de l'inégalité des sexes : des outils et des corps, L'Harmattan, Paris, 1998.

UIT, Déclaration de principes - Construire la société de l'information : un défi mondial pour le nouveau millénaire, UIT, Genève, 2004, Disponible en ligne : <http://www.itu.int/wsis/docs/geneva/ official/dop-fr.html>, consulté le 28 novembre 2013

\section{NOTES}

1. Le terme "Occident " désigne les pays d'Europe de l'Ouest, d'Amérique du Nord ainsi que l'Australie et la Nouvelle-Zélande et leurs socialisations. Ces régions du monde se considèrent elles-mêmes et sont considérées par les organisations internationales, et par une partie des organisations de la société civile au niveau global, comme au " Nord ». C'est dans cette région que se décident globalement les politiques de TIC (l'ensemble des politiques relatives à la réglementation des usages des TIC). Ce «Nord» s'oppose à un "Sud» et, selon Edward Saïd, à l'« Orient " (l'Autre), qui est « une création de l'Occident, son double, son contraire, l'incarnation de ses craintes et de son sentiment de supériorité tout à la fois " (Saïd, 1997, p. 210).

2. L'adjectif « néocolonial » et le substantif « néocolonialisme » décrivent des options politiques des États à la différence des termes postcolonialisme ou post-colonialisme qui signifient une situation historique, postérieure à la colonisation. Ils n'incluent pas uniquement la proclamation politique des indépendances, mais aussi les impacts du colonialisme sur les diasporas, les sociétés plus généralement, les résistances auxquelles ils donnent lieu et à de multiples égards, tant géographiques que culturels et épistémiques.

3. Dans l'ensemble de cet article, le terme «race » est utilisé, entendu comme construction sociale et non donnée biologique entre guillemets. On entend ainsi rendre compte d'une réalité 
objective liée à la discrimination de « race » (au sens large, incluant les Noirs, les Blancs, les métis), comme les Afro-américains peuvent y faire référence (Davis, 1983).

4. La philosophe dissocie le pouvoir de la domination (Arendt, 1961), celle-ci étant entendue comme rapport de commandement et obéissance, basée sur la violence (Arendt, 1972), et celui-là renvoyant à l'expérience de la démocratie.

5. L'ONG Aids Counselling Care and Training (ACCT), créée en 1992 au sein de l'hôpital Chris Hani Baragwanath Hospital à Soweto à Johannesburg et l'organisation Southern Cape Land Committee (SCLC), basée au Cap.

6. Mousso Dambe à Bobo-Dioulasso au Burkina Faso, Nietàa à Ségou au Mali et les Clubs EVF du Pole régional du GEEP à Tambacounda au Sénégal.

7. En mars 2009 au Sénégal et en avril 2009 en Afrique du Sud.

8. Selon deux études de l'agence Portland à Nairobi, publiées successivement en 2012 et 2014, la ville de Johannesburg est la plus active d'Afrique devant Ekurhuleni, toujours en Afrique du Sud, et Le Caire, Durban, Alexandrie. Le Sénégal ne figure pas dans les statistiques. Les utilisateurs sont majoritairement des individus jeunes (entre 20 et 29 ans) - la différenciation par sexe n'est pas mentionnée - qui twittent depuis leurs téléphones mobiles. L'activité se focalise majoritairement sur les marques comme Samsung, Adidas et Magnum (les crèmes glacées). Le football est le sujet le plus discuté, avant la mort de Nelson Mandela (5 décembre 2013), les hashtags politiques étant encore moins courus. Portland, 2012, How Africa Tweets, Nairobi : Portland, <http://www.portland-communications.com/wp-content/uploads/2013/10/HowAfrica-Tweets.pdf>, consulté le 14 septembre 2014 et <http://www.portlandcommunications.com/publications/how-africa-tweets-2014/>, consulté le 14 septembre 2014.

9. Lesbian, Gay, Bisexual and Transsexual.

10. Plateforme d'action de Pékin. Disponible en ligne : <http://www.un.org/french/womenwatch/ followup/beijing5/docs/prgaction.pdf>, dernière consultation le $1^{\mathrm{er}}$ mars 2010.

11. Selon cette étude, la fracture numérique de genre peut se mesurer selon un indicateur synthétique, dit des « $4 \mathrm{C} »$, qui s'articule sur quatre composantes principales, le contrôle, la pertinence des contenus, les capacités, la connectivité. La composante Contrôle témoigne « des disparités de genre en termes de participation à la décision politique, économique, et citoyenne, du degré de préparation pour la prise en compte du genre dans les politiques de TIC, et de la sensibilité des politiques de TIC aux questions de genre ». La composante Contenu témoigne « des dimensions de genre de l'utilisation faite des outils, produits et services de TIC, de la sensibilité aux questions de genre dans les produits de TIC, et de la pertinence des contenus en termes de genre » (enda, 2005, p. 36).

12. D'après Ethnologue, bien que ce chiffre soit difficile à estimer, le nombre de locuteurs anglais dans le monde est passé en 2009 à 5,5\%. Disponible en ligne : <http://www.ethnologue.com/ ethno_docs/distribution.asp?by=size>, consulté le 6 novembre 2012.

13. Union Latine-Funredes 2007, Langues et cultures sur la Toile, Paris : DTIL. Disponible en ligne : <http://dtil.unilat.org/LI/2007/index_fr.htm>, consulté le 6 novembre 2012.

14. De nombreuses études ont mis en lumière les inégalités de genre dans les médias, parmi lesquelles Gender and Media Baseline Study (GMBS), étude conduite en septembre 2002 par l'organisation sud-africaine GenderLinks. Disponible en ligne : <http://www.genderlinks.org.za/ page.php?p_id=236>, consulté le $1^{\mathrm{er}}$ mars 2010.

15. Ibid.

16. Il n'existe pas d'études sur les propriétaires des entreprises de télécommunications, mais si l'on regarde les noms des membres des conseils d'administration de chaque entreprise intervenant en Afrique, comme ailleurs, on peut observer qu'ils sont majoritairement masculins. 17. Ce terme est utilisé par Rowayda pour signifier l'utilisation des textos pour envoyer des messages. 


\section{RÉSUMÉS}

À partir d'un corpus de 31 entretiens semi-directifs et d'observations participantes menés en Afrique du Sud et au Sénégal, d'une analyse des textes institutionnels des organisations internationales et d'une approche théorique approfondie, l'article expose une analyse de la «société de l'information » sous l'angle des rapports de domination qu'elle reproduit ou qu'elle produit. Il souligne l'imbrication de deux institutionnalisations, celle des TIC et du genre, et présente ses impacts sur les mobilisations des organisations de femmes et féministes locales. En s'inspirant des travaux de Spivak, l'analyse met également en évidence en quoi le genre augmente les facteurs de subalternité des femmes africaines. Discutant les analyses de Quijano, il montre que la colonialité est renouvelée et que le virtuel dépolitise le réel. Dans ce contexte et paradoxalement, des pistes de renouvellement d'une épistémologie de la domination s'esquissent.

From a corpus of 31 semi-structured interviews and participant observation conducted in South Africa and Senegal, an institutional analysis and a thorough theoretical approach, the article presents an analysis of the "Information Society" in terms of domination relationships that it reproduces or it produces. It emphasizes the interweaving of two institutionalizations, the ICT and gender ones, and presents its impacts on local feminist and women's organizations mobilization. Drawing on the work of Spivak, the analysis also highlights how gender increases African women's factors of subalternity. Discussing Quijano's analysis, it shows that coloniality is renewed and that the virtual depoliticizes the real. In this context, paradoxically, leads to renew a domination epistemology outline.

\section{INDEX}

Mots-clés : Colonialité, subalternité, féminisme, Afrique, dépolitisation

Keywords : Coloniality, subalternity, feminism, Africa, depoliticization

\section{AUTEUR}

\section{JOELLE PALMIER}

Docteure en Sciences politiques, Chercheure associée au Laboratoire Les Afriques dans le MondeUMR 5115, F-33600 Pessac

joelle.palmieri@gmail.com 